\title{
Cutaneous manifestations of neoplastic disease
}

The skin is a window to systemic disease and malignancies, as it is readily visible. Cutaneous manifestations of internal malignancies include metastases to the skin, paraneoplastic dermatoses, malignancy associated genodermatoses, and dermatoses induced by environmental carcinogens. This article deals with the recognition and diagnosis of paraneoplastic dermatoses.

\section{Paraneoplastic dermatoses}

Paraneoplastic dermatoses are important cutaneous markers of internal malignancy. They form a heterogeneous group of skin conditions, where tumour induced immunological factors, hormones, metabolic disorders, and epidermal growth factors may play a role in their genesis, and frequently indicate the type, localisation and prognosis of the underlying tumour. The course of the tumour and that of the skin manifestation generally run in parallel. When the skin condition and the neoplasm follow a parallel course, the former can be useful in monitoring recurrences of the latter. In some cases cancers may be asymptomatic for years, and skin changes may appear long before the cancer is diagnosed.

Paraneoplastic dermatoses are classified into those associated with solid tumours and those more commonly associated with haematological malignancies. 


\section{Clinical manifestations associated with solid tumours}

\section{Acanthosis nigricans}

This is a symmetrical brown-black pigmentation with velvety thickening commonly in the axillae (figure 1), neck, groin, and popliteal and antecubital fossae. Involvement of extremities, mucosa, mucocutaneous junction of the lips, flexural surface of fingers and palms (tripe palm), presence of generalised pruritus and tylosis are also associated with underlying malignancies. Occasionally malignant acanthosis may become generalised and appear acutely.

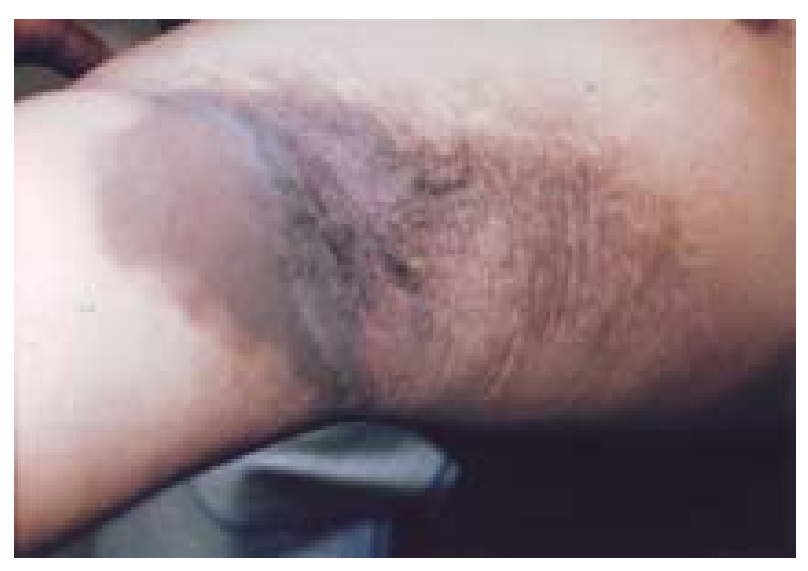

Figure 1. Acanthosis nigricans of the axilla.

In one review of malignant acanthosis $92 \%$ had an abdominal cancer, $65 \%$ of which were gastric, and the remainder were carcinomas of the uterus, liver, intestine, colon, rectum and ovary. Extra-abdominal malignancies included breast, lung and lymphoma. Acanthosis may resolve with eradication of the tumour. Acanthosis palmaris or tripe palm consists of thickened palms with enhanced skin markings, giving rise to a velvety or honeycombed appearance. If acanthosis palmaris occurs alone it is more commonly associated with bronchial carcinoma than with gastrointestinal malignancies.

The exact mechanism by which acanthosis is produced is unknown but it is thought that the tumour secretes a protein which stimulates epidermal hyperproliferation. Acanthosis nigricans occurring with a malignancy or a pituitary adenoma probably results from the secretion of tumour products with insulin-like activity.

\section{Dermatomyositis}

Dermatomyositis comprises the triad of a violaceous rash, proximal myopathy and either elevated serum creatine phosphokinase or an abnormal electromyogram. Cutaneous signs are characteristic with a periorbital heliotrope rash (figure 2), and purplish streaks along the dorsal aspect of the fingers (Gottron's papules). In contrast to autoimmune dermatomyositis, the skin features of malignancy associated dermatomyositis tend to predominate over the myositis. In adults $40 \%$ of dermatomyositis is associated with malignancy of the lung in men, and breast or ovaries in women. Association with nasopharyngeal carcinoma is reported. Usually skin features regress following treatment but relapse of the rash can herald recurrence of the tumour.

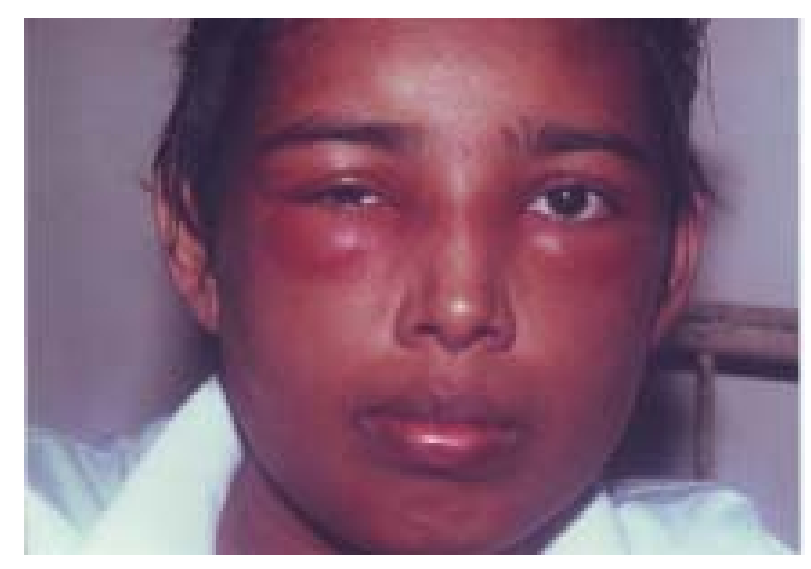

Figure 2. Dermatomyositis periorbital heliotrope rash.

\section{Leser- Trëlat sign}

The rapid eruption of seborrhoeic keratosis (figure 3) with a malignancy is Leser-Trëlat sign. This is associated with adenocarcinoma of the gastrointestinal tract and breast. Rarely it may be associated with malignant melanoma, malignant haemangio-pericytoma, or transitional cell carcinoma of the bladder. Association with underlying malignancy is stronger when it is seen with acanthosis nigricans. Pregnancy and some benign tumours have also been associated with this condition. Patients with this sign associated with malignancy have an average life-span of about 10 months.

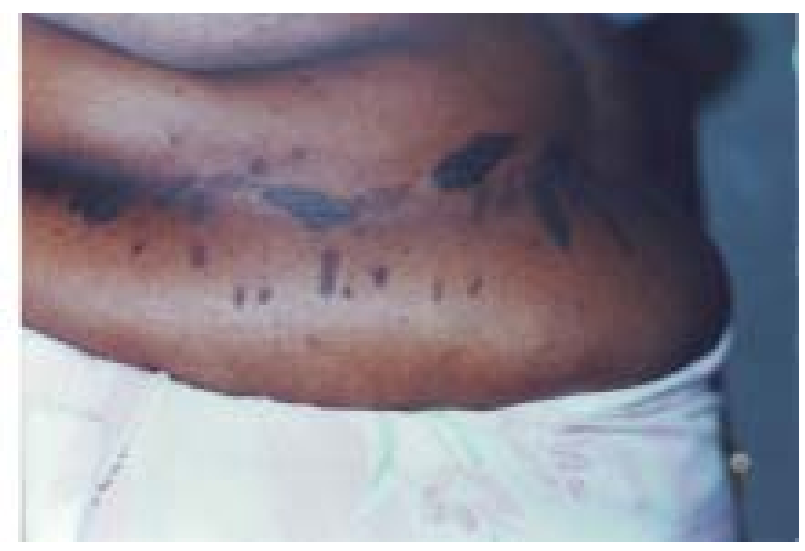

Figure 3. Leser-Trëlat sign: seborrhoeic keratosis over abdomen. 


\section{Necrolytic migratory erythema (glucagonoma syndrome)}

This feature starts with superficially eroding areas of erythema that progress to fragile vesicles and bullae formation. They heal within 7-14 days leaving postinflammatory pigmentation in a polycyclic pattern. Irregular centrifugal spread gives an annular or figurate pattern. They may start at any site but tend to be more pronounced over the lower abdomen, groin, perineum, buttocks and thighs. Mucosal involvement frequently causes glossitis. This skin lesion is a manifestation of glucagonoma. Its course does not follow that of the endocrine syndrome, though surgical treatment or chemotherapy may alleviate the rash. Very rarely this can occur without glucagonoma in chronic liver disease or malabsorption with villous atrophy.

\section{Erythema gyratum repens}

These are bizzarre concentric bands which follow one another in repeated wave forms, often palpable and erythematous, giving rise to a "wood-grain" appearance to the skin. Peripheral scales may be present. The whole body may be affected but the rash is more evident on the trunk, and usually spares the face, hands, and feet. Lesions may be serpiginous and associated with pruritus. A rapidly evolving type is often associated with adenocarcinoma of the lung, breast, stomach, bladder and prostate. The cutaneous features closely mirror the clinical course of the malignancy.

\section{Acquired hypertrichosis languinosa}

This manifestation may precede the diagnosis of the neoplasm by several years, and is common in women between 40 and 70 years. It is characterised by increasing lanugo hair usually on the face within eyebrows and eyelashes. Hair may appear on the trunk, axillae and limbs but sparing the palms, soles and genitals. Other associated features include scleroderma, acanthosis nigricans, glossitis, and papillary hypertrophy of the tongue. Commonly associated malignancies are lung and colorectal cancers. Other associated cancers include ovary, uterus, bladder, gallbladder, pancreas and kidney, and leukaemia and lymphoma.

\section{Paraneoplastic acrokeratosis (Bezex syndrome)}

Cutaneous changes of Bezex syndrome start as a violaceous erythema and scaling on the ears, nose, hands, and feet. Later the lesions become hyperkeratotic on the hands and feet. They may become generalised, often with nail dystrophy and paronychia. Facial lesions may appear more like lupus erythamatosus, whereas acral lesions are psoriasiform. Acrokeratosis is associated with squamous cell carcinoma of the oropharynx, larynx, oesophagus, lung, metastatic adenocarcinoma of the prostate, and transition cell carcinoma of the bladder.

\section{Multicentric reticulohistiocytosis}

This rare condition presents with pink, brown or grey papules and nodules (figure 4) ranging in diameter from a few millimeters to $2 \mathrm{~cm}$ that appear on the hands and may spread to the face. Lesions may appear as pathognomonic "coral beads", and do not ulcerate. Lesions may also appear on the knees, shoulders, hips, elbows, ankles, feet and spine. It is associated with a destructive arthropathy, and the skin lesions are painful. Associated malignancies include myelomonocytic leukaemia, and breast, ovarian, gastric and cervical carcinoma. Association with malignant melanoma, adenocarcinoma of the bowel, penile squamous cell carcinoma and endometrial papillary serous carcinoma has been reported. The course of multicentric reticulohistiocytosis is unpredictable, and does not parallel that of the neoplasm.

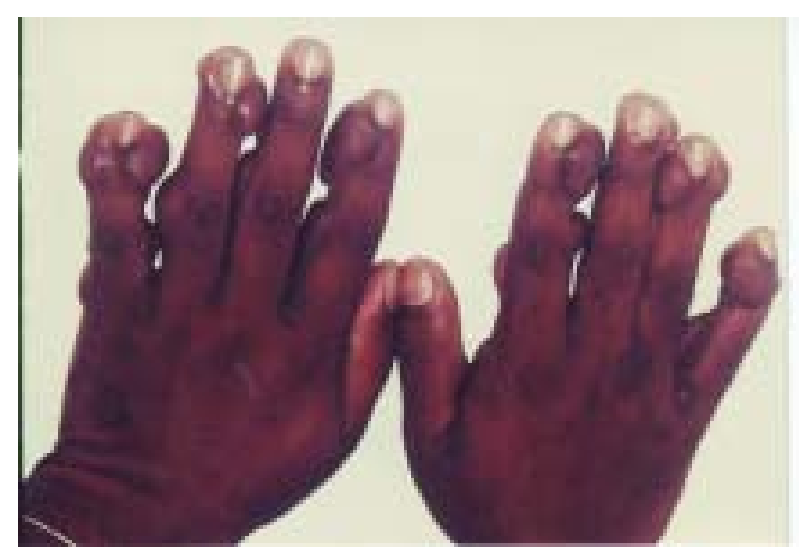

Figure 4. Multicentric reticulohistiocytosis papules and nodules on the hands.

\section{Migratory thrombophlebitis}

This migratory thrombophlebitis is associated with carcinoma of the pancreas, lung and stomach. Lesions are usually recurrent and multiple and a variety of sites, especially the upper and lower extremities, are involved. Intravascular low grade hypercoagulation is implicated as the mechanism.

\section{Scleroderma-like skin changes}

These may be associated with carcinoid syndrome, carcinoma of the oesophagus and rarely with lymphoma of the gut. 


\section{Clinical manifestations associated with haematological malignancies}

\section{Acquired icthyosis}

This resembles autosomal dominant icthyosis (figure 5). It is seen in older persons of both sexes as diffuse, small, white and brown rhomboidal scales with elevated margins. They tend to accumulate on the trunk and extensor surfaces of the limbs. It usually spares the flexural skin folds, palms and soles. Hodgkin lymphoma is reported in $70-80 \%$ of patients with acquired icthyosis. It may occur with non-Hodgkin lymphoma, multiple myeloma, leukaemias and adenocarcinoma of the breast.

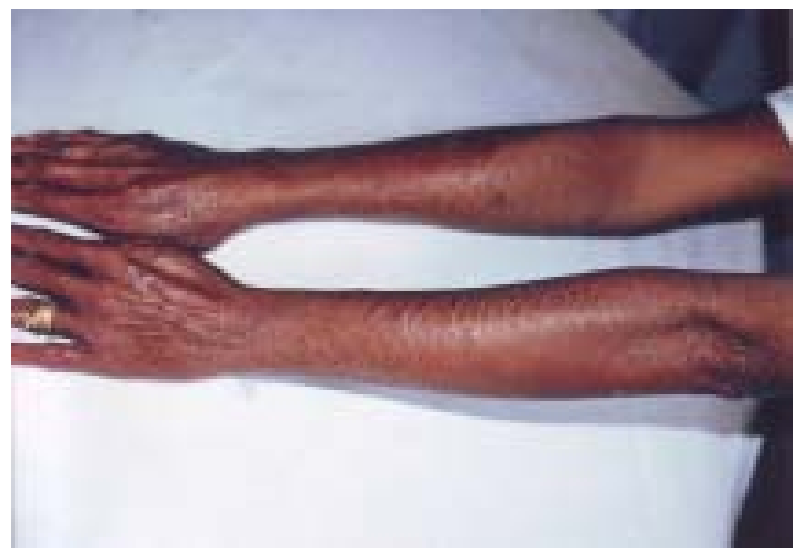

Figure 5. Acquired icthyosis, rhomboidal scales with raised margins.

\section{Pyoderma gangrenosum}

These are as painful nodules or pustules that evolve to an enlarging necrotic ulcer with undermined violaceous borders (figure 6). Lesions which are superficial, appear rapidly, and bullous are associated with myeloma, myeloid leukaemias, lymphoma, myeloid metaplasia, carcinoma of the colon, bladder, prostrate, breast, bronchus, ovary and adrenocortical carcinoma.

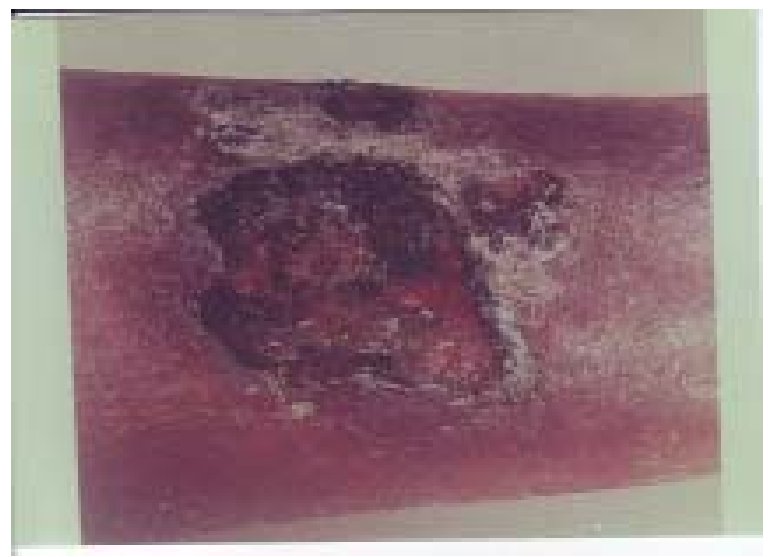

Figure 6. Pyoderma gangrenosum.

\section{Vasculitis}

Vasculitis has been described in association with myeloproloferative disorders and myelodysplastic syndromes.

\section{Sweet syndrome}

The syndrome is characterised by fever, neutrophil leucocytosis, and erythematous tender plaques or nodules (figure 7). Typical skin lesions are red or purple, tender, sharply demarcated plaques whose surface is uneven, with a pseudo-vesiculated appearance. Sweet syndrome responds dramatically to systemic steroids. Common malignancies associated with it are acute myelogenous leukaemia and lymphoma, and rarely solid tumours of the genitourinary tract.

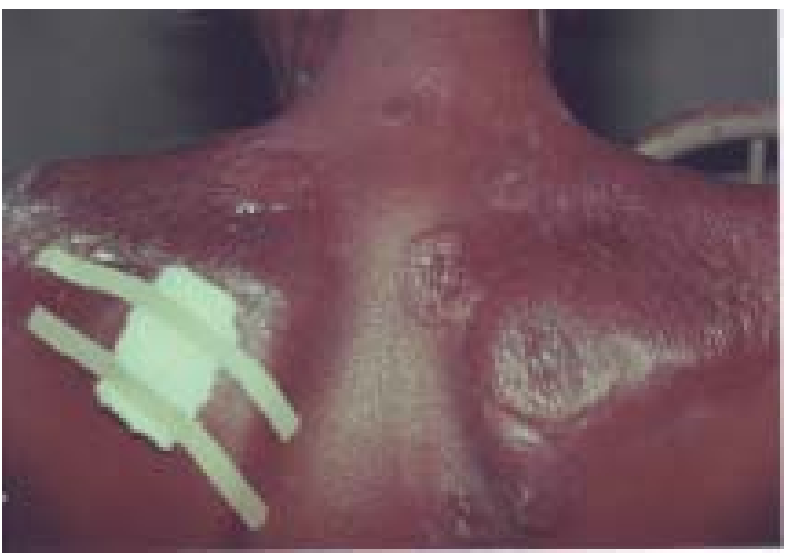

Figure 7. Sweet syndrome. Tender reddish plaques with uneven surface.

\section{Erythema annulare centrifugum}

This is a chronic figurate erythema that begins as red oedematous papules, that enlarge peripherally to form annular, arcuate, or a polycyclic pattern. The lesions are relatively asymptomatic, and are most common on the buttocks, thighs and upper arms. They often disappear within a few weeks, giving rise to fresh lesions. Erythema annulare centrifugum is rarely associated with myeloproliferative disorders.

\section{Paraneoplastic pemphigus}

These lesions are pruritic, polymorphous eruption on the trunk (figure 8) including bullae and erythematous or verrucous target - like papules or plaques. Mucosal involvement is classically prominent, with involvement of conjunctivae and genitalia. Involvement of the palms and soles may be severe. The most commonly associated malignancy is non-Hodgkin lymphoma (42\%), followed by chronic lymphocytic leukaemia (29\%). 


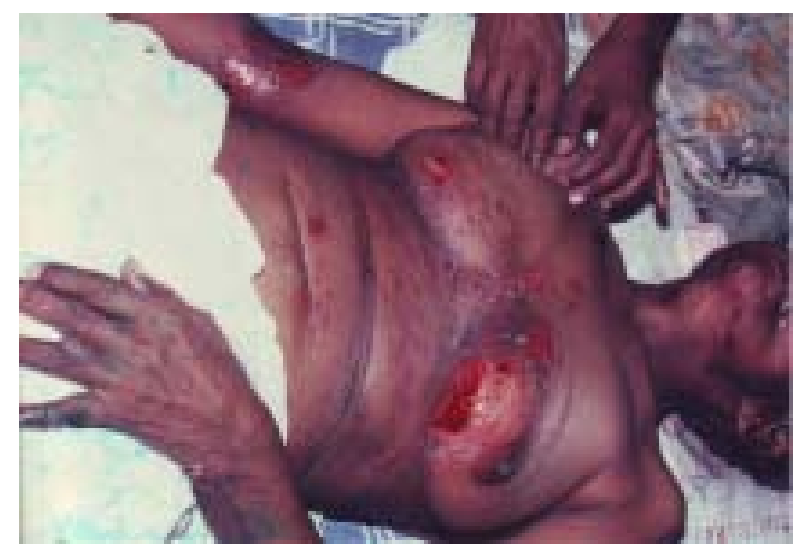

Figure 8. Paraneoplastic pemphigus. pruritic, polymorphous pruritic, polymorphous bullae, and erythematous papules and plaques on the trunk.

\section{Necrobiotic xanthogranuloma}

This presents as multiple sharply demarcated, indurated, non-tender dermal or subcutaneous nodules and plaques. Periorbital involvement is common. Lesions vary from violaceous to red orange, and enlarge to form infiltrative plaques that may show central atrophy. Though most of the lesions are asymptomatic, some may be pruritic or painful. Ulceration and scarring are common. Associated malignancies include multiple myeloma and lymphoproliferative disorders.

\section{Cutaneous amyloidosis}

The most common cutaneous manifestations of this condition are purpura and echymoses over the upper eyelids, face and neck. Other cutaneous manifestations include papules, plaques, nodules, bullae, alopecia and scleroderma. This is usually associated with multiple myeloma.

\section{Further reading}

1. Poole S, Fenske NA. Cutaneous markers of internal malignancy ii paraneoplastic dermatosis and environmental carcinogens. Journal of the American Academy of Dermatology 1993; 28: 147- 64.

2. Vinh Q Chaung, Samuel L, Mashella, et al. Clinical and pathologic findings of paraneoplastic dermatosis. Journal of the American Academy of Dermatology 2006; 54: 745-62.

3. Ragunathan RW. Cutaneous markers of internal malignancy. Galle Medical Journal 2002; 2: 63-70.

4. Higgins EM, du Vivier AW. Cutaneous manifestations of malignancy disease. British Journal of Hospital Medicine 1992; 48: 552-61.

5. Kurzrock R, Cohen PR. Cutaneous paraneoplastic syndromes in solid tumors. Journal of the American Medical Association 1995; 99: 662-71.

R W Ragunathan, Dermatology Unit, Teaching Hospital, Karapitiya, Galle, Sri Lanka.

Correspondence: RW, e-mail: <mkraguna@sltnet.lk>. Competing interests: none declared. 